\title{
Structure of Cuprammonium Regenerated Cellulose Hollow Fiber (BMM Hollow Fiber) for Virus Removal
}

\author{
Takashi Tsurumi, Naoki Osawa, Hidetoshi HitaKa, \\ Tomoko Hirasaki, Kazuhito Yamaguchi, ${ }^{*}$ Sei-ichi Manabe, \\ and Takashi YAMASHIKI \\ BMM Project, Fibers and Textiles Administration, Asahi \\ Chemical Industry Co., Ltd., The Imperial Tower 18F, \\ 1-1 Uchisaiwaicho 1-chome, Chiyoda-ku, \\ Tokyo 100, Japan \\ * Institute of Laboratory Animals, Yamaguchi University \\ School of Medicine, Ube, Yamaguchi 755, Japan
}

(Received December 15, 1989)

\begin{abstract}
An attempt to analyze the pore structure of the cuprammonium regenerated cellulose hollow fiber (BMM hollow fiber) in order to clear up its filtration mechanism was made. The electron microscopy was employed to get the concrete images of the structure. The cellulose particles of rod-like shape with circular cross section having mean diameter of about $50 \mathrm{~nm}$ were its constructing units. The pores were classified into two types, i.e., the pore with the average diameter of about $50 \mathrm{~nm}$ and another with the diameter of several hundreds to several thousands $\mathrm{nm}$. The former was estimated to be the capillary formed among neighboring cellulose particles and the latter to be the void formed as a vacant space which was originated by the phase separation as polymer lean phase. The frequency distribution curve of the void size showed several peaks indicating the occurrence of the boundary breakage between voids originated by the elongation of the fiber in the spinning process. The performances of BMM depends mainly on the existence of capillaries, then BMM with higher ability may be obtained by means of the spinning method which can decrease the occurrence of structure breakage due to the elongation during spinning.

KEY WORDS Regenerated Cellulose / Hollow Fiber/Electron Micrography

/ Membrane Structure / Pore / Virus /
\end{abstract}

Several years ago we developed the cuprammonium regenerated cellulose hollow fiber (BMM hollow fiber) for virus removal. ${ }^{1-4}$ The performance of this hollow fiber was evaluated through many tests and it has proved to have the high rejection rate for HIV (causative agent of AIDS) and HBV (hepatitis B virus) while showing the high permeability for proteins even with high molecular weight such as clotting factor eight and nine.

According to our previous paper, ${ }^{5}$ it was deduced that viruses are caught by BMM hollow fiber through two kinds of mechanism, that is, trapping within "voids" and plugging of "capillaries" and the characteristics of pore structure dominate its performance. The detailed structure of the connection of "voids" and "capillaries" in a membrane should be clarified using direct observation of pores in addition to demonstrating the existence of "voids" and "capillaries" experimentally.

In this article we intend to analyze thoroughly the membrane structure of BMM hollow fiber by means of electron microscopy to elucidate its filtration performance and to get the basic concept for improving its performance. 


\section{EXPERIMENTAL}

\section{Sample Preparation}

BMM hollow fiber; The hollow fibers were prepared from cellulose cuprammonium solution through the microphase separation method under the severe control of the spinning condition. ${ }^{6,7}$ The hollow fibers were bundled into $\mathrm{M}$-module having effective filtration area of $0.03 \mathrm{~m}^{2}$. The BMM hollow fiber used in this experiment had the mean pore size $\left(2 \bar{r}_{\mathrm{f}}\right)$ of $30 \mathrm{~nm}$, the inner diameter of $250 \mu \mathrm{m}$ and the wall thickness of $25 \mu \mathrm{m}$.

\section{Measurement}

(a) Pore Characteristics. The mean pore size $2 \bar{r}_{\mathrm{f}}$ was calculated using eq 1 by putting observed values of the filtration rate of water $J\left(\mathrm{ml} \mathrm{min}^{-1}\right)$, the porosity determined by the apparent density of $\mathrm{BMM} \operatorname{Pr} \rho$, the transmembrane pressure $\Delta P(\mathrm{mmHg})$ and the viscosity of water $\eta$ (centipoise),

$$
2 \bar{r}_{\mathrm{f}}=2.0 \times\left(J \cdot d \cdot \eta / \Delta P \cdot P_{r} \rho\right)^{1 / 2}
$$

(b) Electron Microscopy.

i) Aggregation state of cellulose particles (positive image). BMM hollow fiber was immersed in the mixture of methylmethacrylate, butylmethacrylate and initiator. After polymerization the preparation was sliced into thin section with thickness of several $\mu \mathrm{m}$

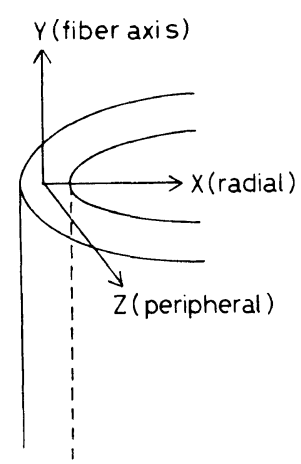

Figure 1. Coordinate of $X, Y$ and $Z$ in the hollow fiber: $X$, axis parallel to the radial direction of the fiber; $Y$, axis parallel to the fiber axis; $Z$, axis parallel to the peripheral direction. through ultramicrotome ("Ultratome III" manufactured by LKB Co., Ltd., Sweden) using diamond knife ("Diatome" manufactured by LKB Co., Ltd., Sweden). The sliced sections were three planes of XY, YZ, and ZX profiles (plane axes of which are parallel to the peripheral direction, radial direction and fiber axis, respectively) shown in Figure 1. The sections were immersed in the solvent of the embedded polymer to wash it out thoroughly and then observed using the scanning electron microscope ("S-570" manufactured by Hitachi Co., Ltd., Japan).

ii) Pore structure made of resin (negative image). The section of $\mathrm{ZX}$ profile (i.e., the plane perpendicular to $Y$ axis) of BMM hollow fiber was prepared just the same way as the positive image memtioned above. The sliced section was immersed in cuprammonium solution to solve cellulose but leave the embedded polymeric resin and observed through the scanning electron microscope ("S-900" manufactured by Hitachi Co., Ltd., Japan).

iii) Frozen fractured image. BMM hollow fiber was fractured along the direction perpendicular to the fiber axis and parallel to $Z$ axis in liquid nitrogen. The fractured sections (nearly parallel to the ZX plane and XY plane) with $\mathrm{Pt}$ coating were observed through the scanning electron microscope ("S-570").

iv) Distribution of pore diameter. The distribution of pore diameter was evaluated from the electron micrograph of the negative image using the graphic data processor ("EO-100" manufactured by Eiko Industrial Co., Ltd., Japan).

\section{RESULTS}

Figures $2 \mathrm{a}$ and $2 \mathrm{~b}$ show the scanning electron micrographs of the frozen fractured sections whose planes are nearly parallel to the ZX plane and XY plane, respectively. In Figure 2a cellulose particles of round shape with various diameters can be seen. But in Figure $2 b$ only 


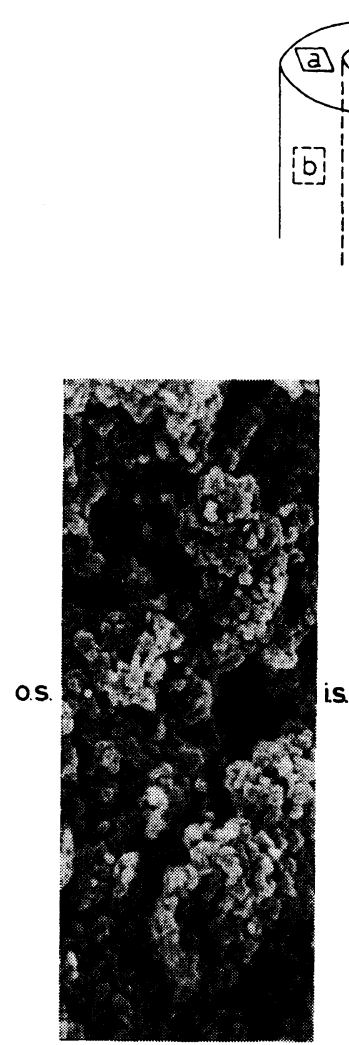

(a)
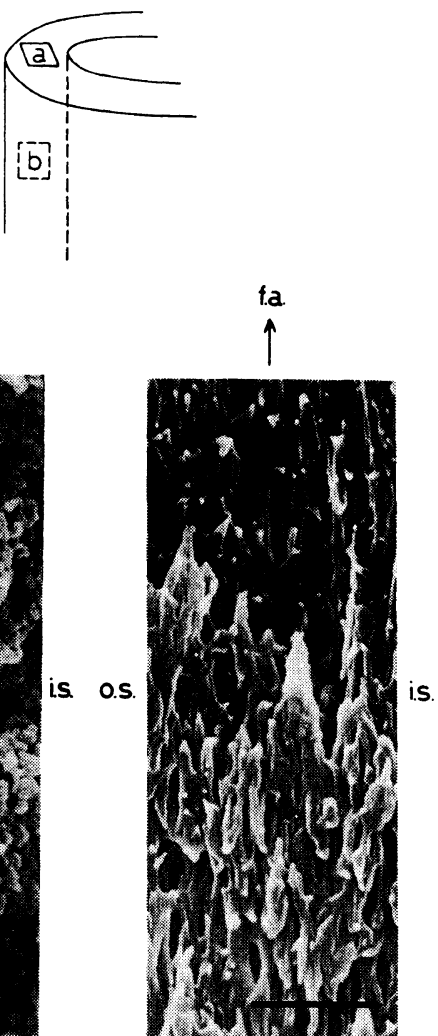

(b)
Figure 2. Scanning electron micrographs of frozenfractured BMM along the direction perpendicular to the fiber axis (a) and to the peripheral direction (b): f.a., fiber axis, i.s., inner surface; o.s., outer surface. Scale bar stands for $1 \mu \mathrm{m}$.

cellulose particles of rod like shape can be seen. So the cellulose particles of rod like shape with the circular cross section are considered to construct the membrane. Figure 3 shows the frequency distribution curve of diameter of cellulose particles observed in Figure 2a. The mean diameter is about $50 \mathrm{~nm}$.

Figure 4 shows the scanning electron micrograph of the $\mathrm{YZ}$ profile. Large oval pores with longer axis parallel to the fiber axis can be observed and small pores are seen scattering in the cellulosic substrate between the large pores. It must be noted that cellulosic substrate are partially fractured as if they were forcibly elongated in the direction of the fiber axis.

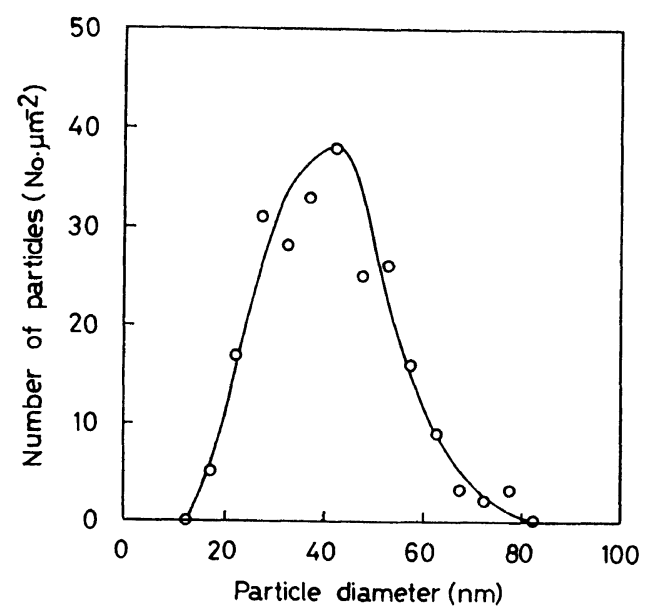

Figure 3. Frequency distribution curve of diameter of cellulose particles constructing BMM hollow fiber.

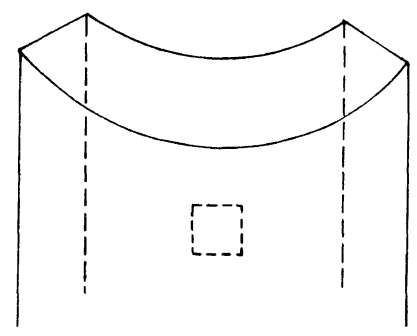

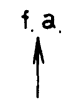

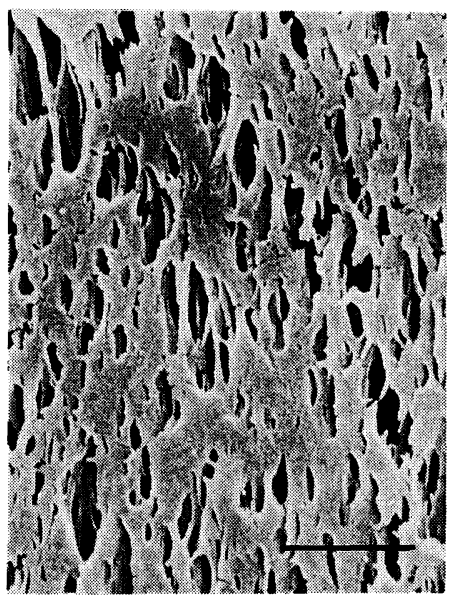

Figure 4. Scanning electron micrograph (positive image) of the section perpendicular to the radial direction (YZ profile) and near the center of BMM hollow fiber: White (or gray) region corresponds to the cellulosic substrate. Scale bar stands for $1 \mu \mathrm{m}$. 


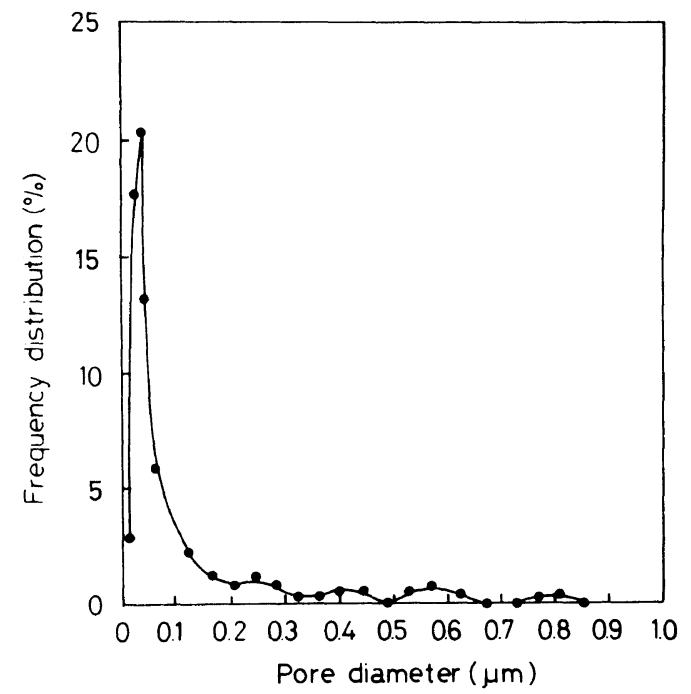

Figure 5. Frequecy distribution curve of pore diameter in YZ profile of BMM hollow fiber obtained from Figure 4.

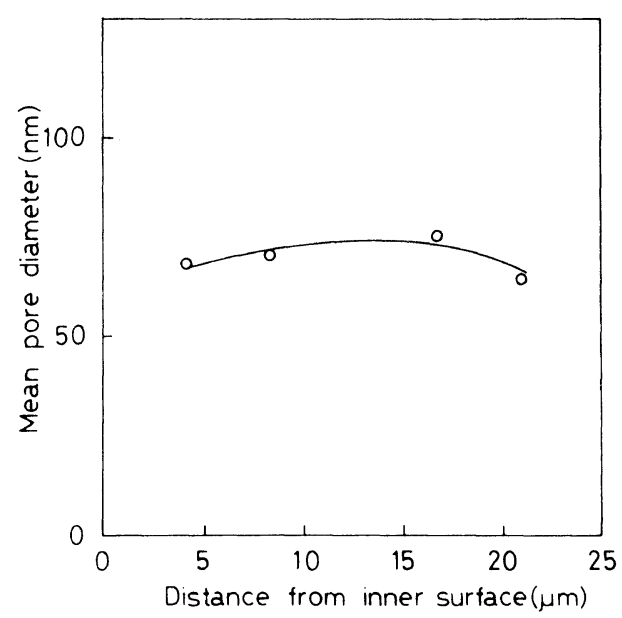

Figure 6. Change in average diameter of each section of $\mathrm{YZ}$ profile as a function of the distance from the inner surface.

Figure 5 shows the distribution of the pore diameter measured from Figure 4 by means of the graphic data processor. There are several peaks in the distribution curve and the peak at the smallest diameter (about $50 \mathrm{~nm}$ ) has the largest frequency. The other peaks locate between about 150 and $500 \mathrm{~nm}$.

Figure 6 shows the change in the mean pore

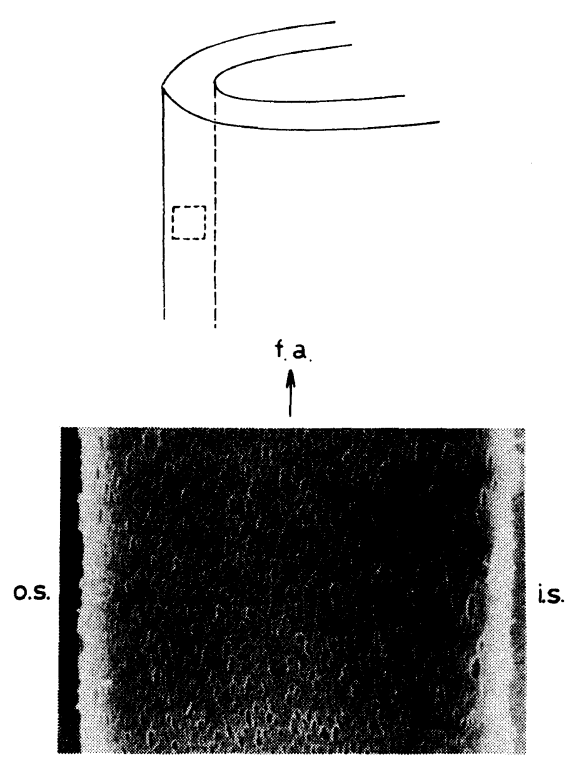

Figure 7. Scanning electron micrograph (positive image) of the section perpendicular to the peripheral direction (XY profile) of BMM hollow fiber: Scale bar stands for $2 \mu \mathrm{m}$.

diameter obtained from each section of $\mathrm{YZ}$ profile as a function of the distance measured from inner surface along the radial direction. The mean diameter keeps constant or changes slightly along the radial direction.

Figure 7 shows the scanning electron micrograph of XY profile. Pores having several hundreds $\mathrm{nm}$ of width and elipsoidal shape whose longer axis is parallel to the fiber axis (f.a.) of BMM hollow fiber and small pores scattering in the cellulosic substrate having several tens nm diameter are observed in Figure 7. Their sizes and shape are similar to those in Figure 4.

Figure 8 shows the scanning electron micrograph of ZX profile. Pores with several thousands to hundreds $\mathrm{nm}$ have various irregular shape which is definately different from those of Figures 4 and 7. Smaller pores having diameter of several tens $\mathrm{nm}$ are also observed.

Judging from the results of Figures 4, 7, and 8 , we can notice that the pore structure of 

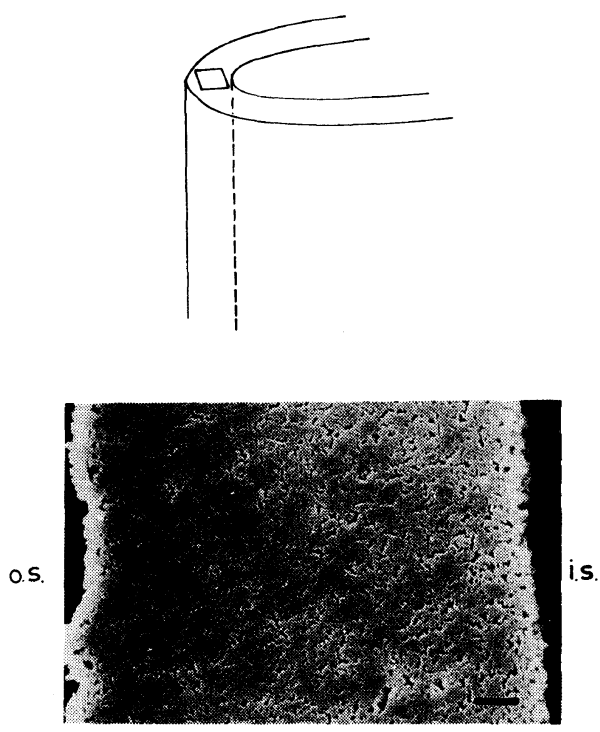

Figure 8. Scanning electron micrograph ([ositive image) of the section perpendicular to the fiber axis (ZX profile) of BMM hollow fiber: Scale bar stands for $2 \mu \mathrm{m}$.

BMM hollow fiber is quasi-isotropic around the fiber axis but anisotropic around the radial and peripheral axes of the hollow fiber.

Figure 9 shows the electron micrograph of the fine structure of the resin left in the pores after dissolving cellulose out from the membrane embeded with the resin. The resin constructs the three-dimensional network in which resin blocks are connected by thin rods.

\section{DISCUSSION}

The existence of cellulose particles in BMM hollow fiber as is obvious in Figure 2 indicates that the hollow fiber is prepared through microphase separation method. By taking into account the results of Figure 4, we can modify Kamide and Manabe's proposal ${ }^{8}$ for formation of pores in a membrane. Figure 10 shows the schematic representation of formation of pores in BMM hollow fiber. This model is based on their proposal and modified in two major points, that is, heterogeneity in the particle size and the anisotropic deformation of cellulose particles to the direction of fiber axis. These
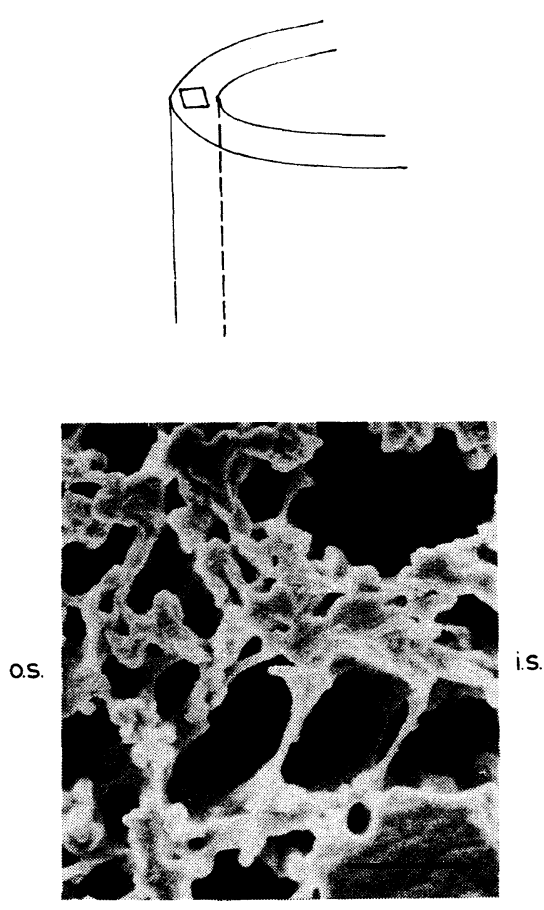

Figure 9. Scanning electron micrograph (negative image) of ZX profile of BMM hollow fiber: White (or gray) region corresponds to the pore itself. Scale bar stands for $500 \mathrm{~nm}$.

two points are originated from the preparation condition of hollow fiber due to the tension of taking up and the short time of the process comparing with the case of the plane type membrane.

Although the structure of BMM hollow fiber observed through the electron microscope has slightly different characteristics from the theoretical ideal membrane ${ }^{9}$ prepared through the microphase separation method, the basic principle that the membrane is composed of polymer particles generated through the microphase separation, holds. Consequently, in order to analyze the pore structure of BMM hollow fiber, we can employ the conceptual model shown in Figure 11, the main part of which is originally proposed by Manabe et al. ${ }^{9}$ for the plane type membrane, although small modification is added to the original model.

According to this model we can deduce that BMM hollow fiber has two types of pores, one 


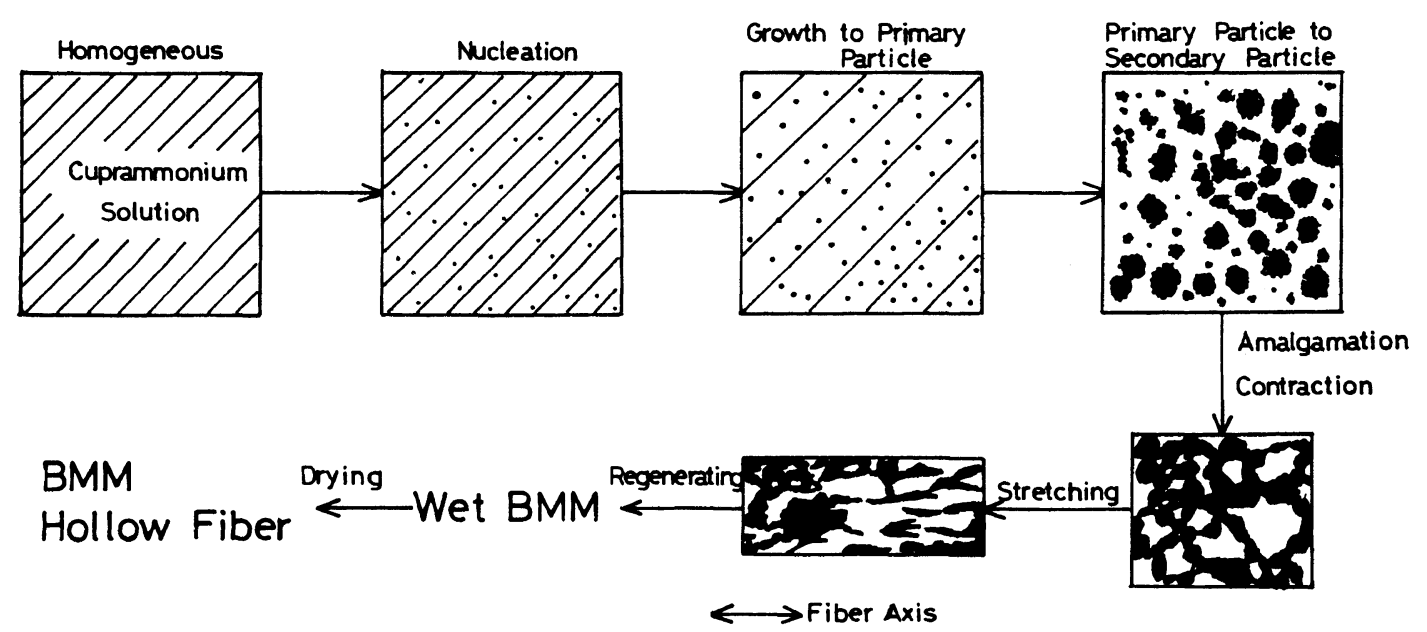

Figure 10. Schematic representation of the formation of pores according to the Kamide and Manabe's proposal $^{8}$ with small modification for BMM hollow fiber: Hatched space stands for the solution before phase separation, filled circles or spots for cellulose rich phase after the phase separation resulting in cellulose particles of the finished BMM hollow fiber and white space for the cellulose lean phase resulting in pores of the finished BMM hollow fiber.

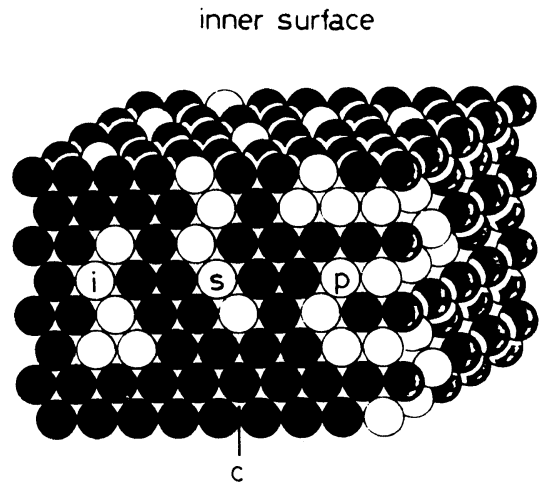

outer surface

Figure 11. schematic representation of pore structure of membrane made through microphase separation with small modification of the Manabe et al.'s model $^{9}$ for BMM hollow fiber: Filled spheres and vacant spheres indicate cellulose particles and vacant space, respectively. $i$, isolated voids; $p$, penetrating voids; s, semi-open voids; c, capillary.

is the pore formed among neighboring secondary particles (this pore is referred to as a "capillary") and the other is the pore formed by vacant particles or aggregates of them (this pore is referred to as a "void"). The capillaries are considered to be very narrow and construct channels linking themselves through out the whole thickness of the membrane. On the other hand the voids may be classified into three types, that is, isolated voids which are connected to other voids only by capillaries, semiopen voids which construct channels open to only one surface of the membrane and penetrating voids which construct the "channel" penetrating the membrane from the inner surface to the outer surface.

The BMM hollow fiber is composed of rod-like cellulose particles having circular cross section with the diameter of about $50 \mathrm{~nm}$. These cellulose particles are considered as the secondary particles which were elongated in the spinning process (see Figure 10). The large pores with the diameter of several hundreds $\mathrm{nm}$ seen in Figure $2 \mathrm{~b}$ among cellulose rods are considered to correspond to the large oval pores observed in Figures 4 and 7, the large pores with various kinds of shape observed in Figure 8 and the resin blocks observed in Figure 9. The peaks located between about 150 and $500 \mathrm{~nm}$ in the distribution curve in Figure 5 may also correspond to these large pores, that is, voids shown in Figure 11. On the other hand, small pores with the diameter around $50 \mathrm{~nm}$ 
are considered to correspond to the small pores scattering in the cellulosic substrate observed in Figures 4, 7, and 8, and the thin rods observed in Figure 9. The highest peak in Figure 5 may also correspond to these small pores in their size and population. It will be apparent that these small pores are capillaries shown in Figure 11 taking into accout their size, shape and existing population.

Figure 12 is the schema of the pore structure
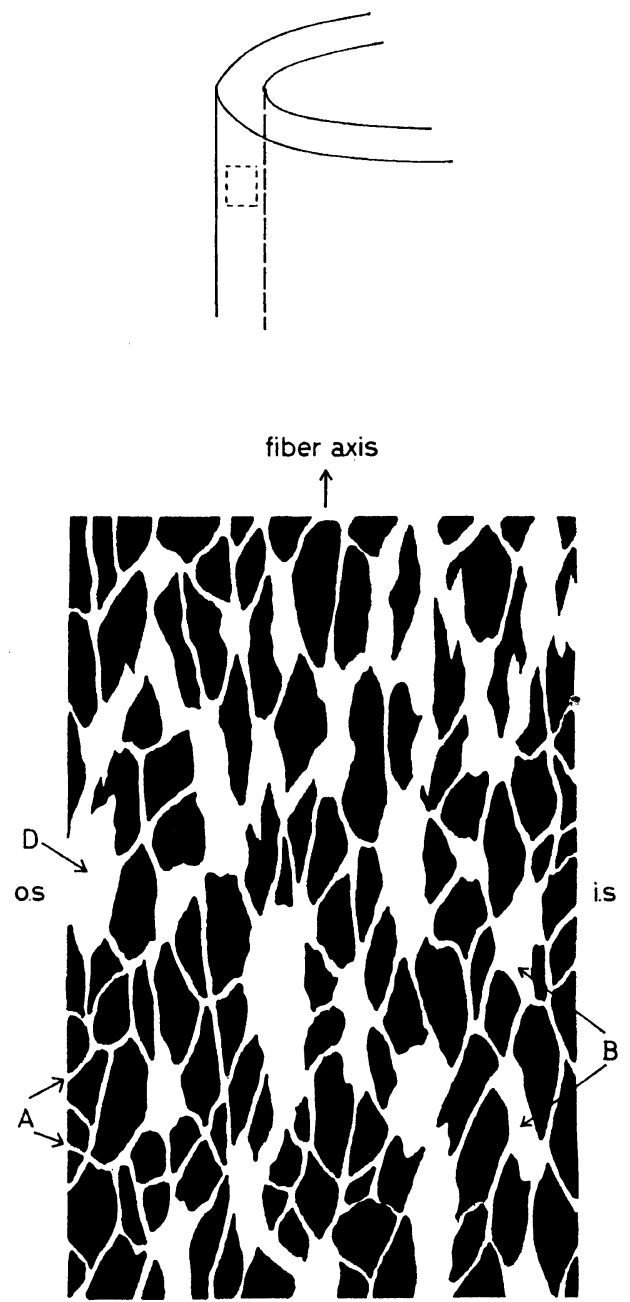

Figure 12. Schematic representation of pore structure of ZX profile of BMM hollow fiber: Black blocks stand for the aggregates of the secondary particles of cellulose and white zone stands for pore channel. Scale bar stands for $500 \mathrm{~nm}$. of BMM based on information obtained from the electron micrographs in Figures $2 a, 2 b, 4$, 7,8 , and 9 and the above discussion. In this schema narrow passages are "capillaries" and wide spaces are "voids." When the protein solution containing virus particles with the diameter larger than that of the "capillaries" are feeded to the membrane with the pore structure shown in Figure 12, small protein molecules pass freely through "capillaries" and "voids" although the virus particles easily plug the "capillaries" and might pass the membrane only through the channels penetrating the membrane from the inner surface to the outer surface. Judging from the fact that BMM hollow fiber has both high rejection rate for virus and high permeability for proteins, "capillaries" and "voids" are considered to be arranged in series connection in the membrane. It may be reduced from the structural analysis mentioned above, that if the membrane has the "capillaries" with the diameter larger than that of proteins and smaller than that of viruses and at the same time does not have the channels

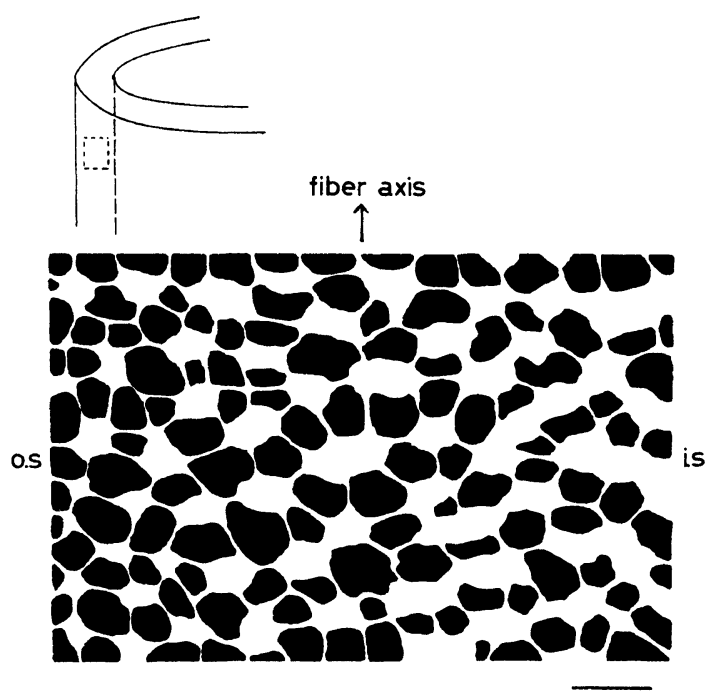

Figure 13. Schematic representation of pore structure of ZX profile of ideal BMM hollow fiber which would be obtained in the imaginary spinning process under no-stretching condition: Black blocks and white zone indicate the same as in Figure 12. Scale bar stands for $500 \mathrm{~nm}$. 
formed by voids which penetrate the membrane, it will exert higher performances.

The structural characteristics of BMM hollow fiber deduced from the electron micrographs are the elongated pores (see Figure 12) and the elongated cellulosic particles (see Figure 10) in the direction parallel to the fiber axis. This elongation may be due to the force working on the hollow fiber in the longitudinal direction during spinning. The oval shape of "voids" and cellulose structures partially fractured mainly perpendicular to the fiber axis may be one of the products of stretching. The fracture of the cellulosic substrate may lead to the breakage of the interfacial wall between "voids" and cause the linkage of these voids resulting in the formation of channels penetrating the whole thickness of the membrane. Then, if it becomes possible to reduce the rate of stretching during spinning, the formation of the penetrating channels by stretching can be avoided and the higher performance will be obtained.

Fgure 13 shows the schematic representation of the structure of the ideal BMM which would be obtained in the spinning process without stretch. In this membrane the channels formed by voids $(C)$ are located in certain amount near the inner surface. Such channels are indispensable to avoid plugging of pores with large particles such as virus and proteins with large molecular weight. But the number of such channels gradually decrease toward the center of the membrane and finally disappear near the outer surface, where all voids should be isolated ones (B) to avoid the leak of virus. The pore structure near the outer surface resembles the connection of nerve cells (neuron) and can be called therefore "neuronic capillary-void structure."

\section{REFERENCES}

1. K. Okuyama, Y. Fukushima, Y. Miura, R. Honma, S. Manabe, G. Ishikawa, M. Satani, and K. Komuro, J. J. Clinical Hematology, 29, 662 (1988).

2. Y. Hamamoto, S. Harada, S. Kobayashi, K. Yamaguchi, H. Iijima, S. Manabe, T. Tsurumi, H. Aizawa, and N. Yamamoto, Vox Sang., 56, 230 (1989).

3. S. Manabe, T. Tsurumi, G. Ishikawa, M. Satani, T. Yamashiki, Y. Hamamoto, K. Yamaguchi, S. Kobayashi, and N. Yamamoto, Membrane, 14(1), 77 (1989).

4. S. Sekiguchi, K. Ito, M. Kobayashi, H. Ikeda, T. Tsurumi, G. Ishikawa, S. Manabe, M. Satami, and T. Yamashiki, Membrane, 14(4), 101 (1989).

5. T. Tsurumi, N. Osawa, T. Hirasaki, K. Yamaguchi, S. Manabe, and T. Yamashiki, Polym. J., 22, 304 (1990).

6. S. Manabe, M. Iwata, and M. Inoue, Japanese Patent Application, 58-89625 (1983).

7. M. Iwata, S. Manabe, and M. Inoue, Japanese Patent Application, 59-204911 (1984).

8. K. Kamide and S. Manabe, "Materials Science of Synthetic Membranes," D. R. Lloyd, Ed., American Chemical Society, Washington, D.C., 1985, p 197.

9. S. Manabe, Y. Kamata, H. Iijima, and K. Kamide, Polym. J., 19, 319 (1987). 